\title{
4
}

\section{Titanium Alloys at Extreme Pressure Conditions}

\author{
Nenad Velisavljevic ${ }^{1}$, Simon MacLeod ${ }^{2}$ and Hyunchae Cynn ${ }^{3}$ \\ ${ }^{1}$ Los Alamos National Laboratory \\ ${ }^{2}$ Atomic Weapons Establishment \\ ${ }^{3}$ Lawrence Livermore National Laboratory \\ 1,3 USA \\ ${ }^{2} U K$
}

\section{Introduction}

The electronic structures of the early transition metals are characterised by the relationship that exists between the occupied narrow $d$ bands and the broad $s p$ bands. Under pressure, the $s p$ bands rise faster in energy, causing electrons to be transferred to the $d$ bands (Gupta et al., 2008). This process is known as the $s-d$ transition and it governs the structural properties of the transition metals.

At ambient conditions, pure Ti crystallizes in the 2-atom hcp, or $\alpha$ phase crystal structure (space group $\mathrm{P}_{3} / \mathrm{mmc}$ ) and has an axial ratio $(c / a) \sim 1.58$. Under pressure, the $\alpha$ phase undergoes a martensitic transformation at room temperature (RT) into the 3 -atom hexagonal, or $\omega$ phase structure (space group P6/mmm). The appearance of the $\omega$ phase at high pressure raises a number of scientific and engineering issues mainly because the $\omega$ phase appears to be fairly brittle compared with the $\alpha$ phase, and this may significantly limit the use of $\mathrm{Ti}$ in high pressure applications. Furthermore, after pressure treatment the $\omega$ phase appears to be fully, or at least, partially recoverable at ambient conditions, thus raising questions as to which is the lowest thermodynamically stable crystallographic phase of $\mathrm{Ti}$ at $\mathrm{RT}$ and pressure.

This chapter deals with the behavior of $\mathrm{Ti}$ alloys under extreme pressure and temperature conditions. Our recent results are presented and comparison is made to data available in the open literature. We volume compressed Ti-6Al-4V (an $\alpha$ alloy) and Ti-Beta-21S (a $\beta$ alloy) in a series of RT diamond anvil cell (DAC) angle-dispersive X-ray diffraction (ADXD) experiments, to investigate the effects of alloying and the pressure environment on phase stability and the transformation pathway. However, before describing the results of these experiments in detail, we present a brief review of the current state of knowledge of $\mathrm{Ti}$ at high pressure.

\section{Ti at high pressure}

The phase relations of Ti have been studied extensively at high pressure. As indicated in the introduction, there is great interest in the properties of $\mathrm{Ti}$ at high pressure and temperature. 
The RT $\alpha \rightarrow \omega$ phase transition has been observed to occur between $2 \mathrm{GPa}$ and $12 \mathrm{GPa}$, depending on the experimental technique, the pressure environment, and the sample purity. Although most of the recent work has involved the use of diamond anvil cells (DACs) to volume compress $\mathrm{Ti}$ in the static regime (for example, Ming et al., 1981; Akahama et al., 2001; Vohra et al., 2001; Errandonea et al., 2005), many studies have also utilised large volume presses to statically compress Ti (Jamieson 1963; Jayaraman et al., 1963; Bundy 1963; Bundy 1965; Zhang et al., 2008), and to a lesser extent, shock techniques have been employed to compress $\mathrm{Ti}$ in the dynamic regime (see Gray et al., 1993; Trunin et al., 1999; Cerreta et al., 2006).

\subsection{Dynamic compression of $\mathrm{Ti}$}

The effects oxygen and other interstitial impurities on $\omega$ phase formation in $\mathrm{Ti}$ has been studied using shock compression (Gray et al., 1993 \& Cerreta et al., 2006). In these studies, samples with oxygen content 360 ppm (high purity Ti) and 3700 ppm (A-70) were shocked whilst simultaneously probed using a real-time velocity interferometer system for any reflector (VISAR) diagnostic and then analysed post-shock. A phase transition was reported at 10.4 GPa for the high purity sample, whereas the A-70 sample did not show any evidence of a phase change up to 35 GPa (Gray et al., 1993 \& Cerreta et al., 2006). A post shock (11 GPa) recovered high purity sample retained $28 \%$ of the $\omega$ phase (Gray et al., 1993). The suppression of the $\alpha \rightarrow \omega$ stress transformation in Ti is likely caused by the presence of interstitial oxygen (Cerreta et al., 2006). Greef et al. performed an analysis of early Ti shock measurements, but were unable to allow for sample purity in their study, since the oxygen content was not measured in these early experiments, and as a consequence placed the $\alpha \rightarrow$ $\omega$ phase transition at $~ 12$ GPa (Greef et al., 2001).

\subsection{Static compression of $\mathrm{Ti}$}

\subsubsection{Room temperature compression}

Using DACs and X-ray diffraction, the experimental technique RT $\alpha \rightarrow \omega$ phase transition in commercially available purity Ti has been observed to occur between $2 \mathrm{GPa}$ and $12 \mathrm{GPa}-$ in cases where data was collected during pressure release large transformation pressure hysteresis is observed and in some cases the $\omega$ phase can be recovered at ambient conditions (Vohra et al., 1977; Ming et al., 1981; Vohra et al., 2001; Akahama et al., 2001; Errandonea et al., 2005). The effect of the pressure environment (and therefore uniaxial stress) on the $\alpha \rightarrow$ $\omega$ transformation in Ti was studied in a series of DAC and angle dispersive X-ray diffraction (ADXD) experiments (Errandonea et al., 2005). By embedding Ti samples in a variety of pressure-transmitting-media (PTM), Errandonea et al were able to demonstrate that the pressure at which the Ti transformed into the $\omega$ phase was found to increase with an increase in the hydrostaticity of the PTM. For an argon PTM (the most hydrostatic PTM used in the experiment), the $\alpha \rightarrow \omega$ transition occurred in the pressure range between 10.5 GPa and 14.9 GPa, and for the least hydrostatic environment (that is, no PTM), the $\alpha \rightarrow \omega$ transition occurred under pressure between 4.9 GPa and 12.4 GPa (Errandonea et al., 2005). A coexistence of the $\alpha$ and $\omega$ phases over a largish pressure range was also observed, in agreement with earlier findings (Ming et al., 1981). 
Vohra et al. investigated the effects of the impurity levels of oxygen on the $\alpha \rightarrow \omega$ transition (Vohra et al., 1977). In this non-hydrostatic DAC study (no PTM), the oxygen content of the Ti samples was varied between 785 ppm and 3800 ppm (by weight) and the corresponding transition pressure was measured between $2.9 \mathrm{GPa}$ and $6.0 \mathrm{GPa}$.

Ti has been volume compressed at RT (no PTM) in a DAC to a pressure of $220 \mathrm{GPa}$ and found to follow the transformation pathway $\alpha \rightarrow \omega \rightarrow \gamma \rightarrow \delta$ (Vohra \& Spencer, 2001; Akahama et al., 2001). The $\omega$ phase structure was observed to be stable to pressures greater than $100 \mathrm{GPa}$. The reported intermediate orthorhombic $\gamma$ phase (transforming between $\sim 116$ $\mathrm{GPa}-128 \mathrm{GPa}$ ) possessed a distorted hcp crystal structure (space group $\mathrm{Cmcm}$ ), and the orthorhombic $\delta$ phase (transforming at $\sim 145 \mathrm{GPa}$ ) was of a distorted bcc type structure (space group $\mathrm{Cmcm}$ ) (Akahama et al., 2001). These observations for $\mathrm{Ti}$ are at variance with the other Titanium Group transition metals $\mathrm{Zr}$ and Hf, which have been observed to follow the $\alpha \rightarrow \omega \rightarrow \beta$ pathway at RT (Jayaraman et al., 1963; Xia et al., 1990a; Xia et al., 1990b). However, Ahuja et al. have observed the coexistence of a bcc-like structure (referred to by the authors as $\beta^{\prime}$ ) with the $\omega$ phase, during the compression of Ti at RT from $40 \mathrm{GPa}$ to 80 GPa (Ahuja et al., 2004). In this study, Ti was embedded in a $\mathrm{NaCl}$ PTM. The subsequent ADXD patterns could only be fully analysed on the assumption that the additional reflections present in the patterns belonged to the $\beta$ phase of Ti. Laser heating Ti to between $1200 \mathrm{~K}$ and $1300 \mathrm{~K}$ at 78-80 GPa resulted in the formation of a new orthorhombic $\eta$-phase (space group Fmmm), which on decompression, at RT, transformed back into the $\beta^{\prime}$ phase below $40 \mathrm{GPa}$ (Ahuja et al., 2004). On further decompression below $30 \mathrm{GPa}$, the $\beta^{\prime}$ phase reverted back into the $\omega$ phase, and this phase could be quenched to ambient conditions (Ahuja et al., 2004).

Most recently, nano-Ti sample (with grain sizes $\sim 100 \mathrm{~nm}$ ) was compressed at RT in a DAC to $161 \mathrm{GPa}$ and the transformation pathway $\alpha \rightarrow \omega \rightarrow \gamma \rightarrow \delta$ was observed (Velisavljevic et al., n.d.). The slightly high $\alpha \rightarrow \omega$ transition pressure of $10 \mathrm{GPa}$ observed (no PTM), may have been caused by the increase in interface to volume ratio, resulting from the reduction in grain size, and leading to increased resistance to shear deformation. Compared to coarse grained $\mathrm{Ti}$, in nano-Ti there may also have been a larger concentration of interstitial impurities near the grain boundaries, which have been shown to help suppress the $\alpha \rightarrow \omega$ structural phase transition (see Hennig et al., 2005). The $\omega$ phase was observed to be stable up to $\sim 120 \mathrm{GPa}$, and under compression to $127 \mathrm{GPa}$ resulted in a phase transformation to the orthorhombic $\gamma$ phase. A further compression resulted in a transition from $\gamma$ to the $\delta$ phase at $140 \mathrm{GPa}$, in good agreement with a previous study (Akahama et al., 2001). The $\delta$ phase was stable up to 161 GPa. Figure 1 shows a stacked plot of nano-Ti ADXD patterns in the $\omega, \gamma$ and $\delta$ phases (Velisavljevic et al., n.d.). The metastable $\omega$ phase was recovered after pressure treatment, which is consistent with reports from other experiments on $\mathrm{Ti}$, indicating that after pressure release, samples were either recovered in the $\omega$ phase or as a mixture of $\alpha+\omega$ (see Errandonea et al., 2005 \& Vohra et al., 2001). In the case of these nano-Ti experiments, the recovered sample was observed to consist of only the $\omega$ phase (Velisavljevic, n.d.). The high pressure behavior of nano-Ti, including the $\alpha \rightarrow \omega \rightarrow \gamma \rightarrow \delta$ structural phase sequence (without the appearance of the $\beta$ phase), the change in axial $c / a$ ratio with pressure, recovery of $\omega$ phase, and change in volume with pressure and the EOS values (Velisavljevic, n.d.), is very similar and consistent with previous experimental results on Ti (Akahama et al., 2001; Vohra et al., 2001; Errandonea et al., 2005;). 


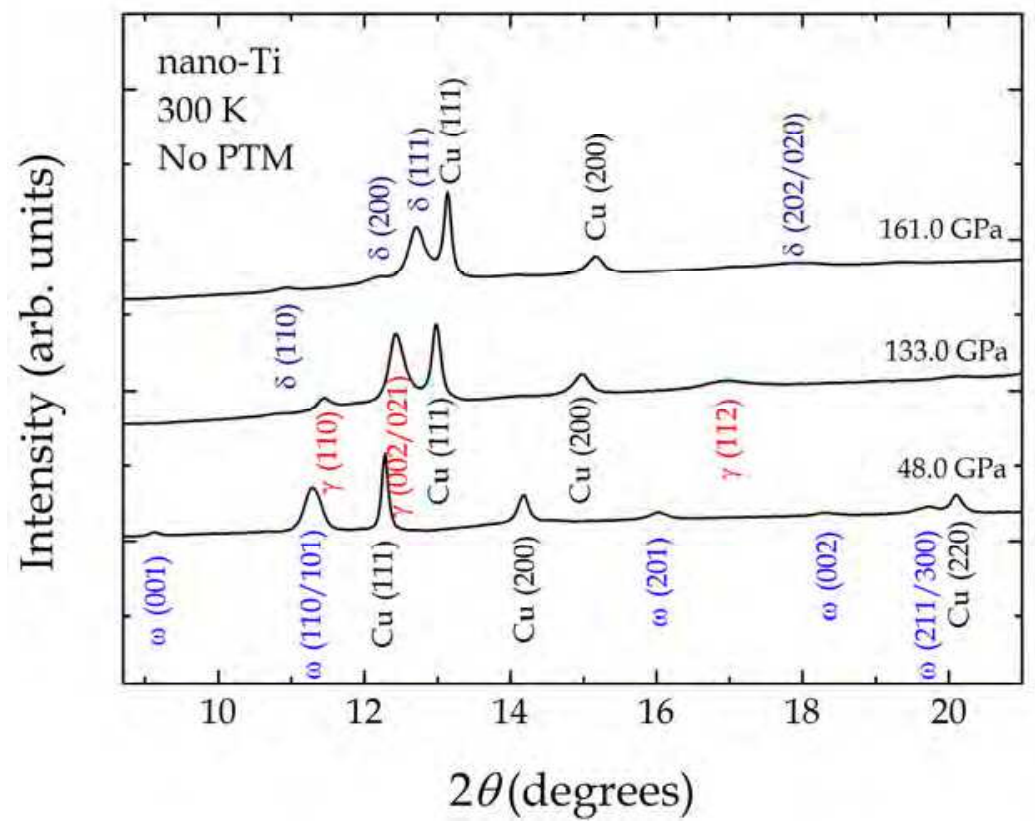

Fig. 1. A stack of ADXD patterns showing pressure induced structural phase transformation $\omega \rightarrow \gamma \rightarrow \delta$ in nano-Ti (Velisavljevic et al., n.d.).

\subsubsection{High temperature compression}

Very few combined static high-pressure and high-temperature studies have been reported for Ti. Thermally treating $\mathrm{Ti}$ to temperatures exceeding $1155 \mathrm{~K}$ at room pressure (RP) transforms the $\alpha$ phase into the more densely packed bcc or $\beta$ phase (space group $\operatorname{Im} 3 \mathrm{~m}$ ), without the intermediate $\omega$ or other crystallographic phase occurring.

Jayaraman et al. studied the $\alpha \rightarrow \beta$ boundary up to $\sim 6.5 \mathrm{GPa}$ and $1100 \mathrm{~K}$, and found that increasing the pressure lowered the $\alpha \rightarrow \beta$ transition temperature (Jayaraman et al., 1963). A more extensive study conducted by Bundy, in which Ti was compressed to $16 \mathrm{GPa}$ and heated to $1200 \mathrm{~K}$ revealed the location of the boundaries of the three solid phases, $\alpha, \beta$ and $\omega$ (Bundy, 1963 \& Bundy, 1965). More recently, Ti was compressed in a cubic anvil apparatus to $8.7 \mathrm{GPa}$ and heated to $973 \mathrm{~K}$ (Zhang et al., 2008). The ADXD patterns collected confirmed the high-temperature phase diagram reported by Bundy. The $\alpha-\omega-\beta$ triple point was estimated at 7.5 GPa and $913 \mathrm{~K}$ (Zhang et al., 2008), in agreement with the previous estimate of $8.0 \pm 0.7 \mathrm{GPa}$ and $913 \pm 50 \mathrm{~K}$ (Bundy, 1965). Zhang et al. observed the $\alpha$-phase to undergo an isotropic compression between RP and $7.8 \mathrm{GPa}$, resulting in the axial ratio $(c / a)$ being constant (1.587) over this pressure range (Zhang et al., 2008).

Errandonea et al. melted Ti in a DAC up to 80 GPa using single-sided laser-heating and the "speckle technique" to determine the onset of melting (Errandonea et al., 2001). No X-ray diffraction patterns were collected in this lab-based study, and so the authors were unable to discriminate between melting from either the $\beta$ phase or the $\omega$ phase. Short term laser- 
heating appeared to lower the $\alpha \rightarrow \omega$ transition pressure in pure $\mathrm{T}$, as was determined in a follow up DAC study conducted by Errandonea at a synchrotron (Errandonea et al., 2005). At $5 \mathrm{GPa}$, Ti was transformed into the $\beta$ phase by laser-heating to $1750 \mathrm{~K}$ and $2150 \mathrm{~K}$ (above melting), at which point the samples were quenched. A mixture of $\alpha$ and $\omega$ phases were obtained at a pressure for which only the $\alpha$ phase existed previously at RT, suggesting that thermal fluctuations may have a similar effect on the $\alpha \rightarrow \omega$ transformation as uniaxial stress.

\subsection{Theoretical treatments of Ti at high pressure}

Although Ti has received much theoretical attention over the years, we will mention here only studies that are of direct relevance to this chapter. First-principles calculations have been used to generate the phase diagram of Ti (Trinkle et al., 2003; Pecker et al., 2005; Hennig et al., 2005; Trinkle et al., 2005; Verma et al., 2007; Hennig et al., 2008; Mei et al., 2009; Hu et al., 2010).

A multiphase equation of state (EOS) of the three solid phases $(\alpha, \beta$ and $\omega)$, the liquid phase and gas phase, was calculated up to $100 \mathrm{GPa}$ and predicted (based on experiment) the $\omega-\beta-$ liquid triple point at around $45 \mathrm{GPa}$ and $2200 \mathrm{~K}$ (Pecker et al., 2005). Verma et al. predicted the $\omega \rightarrow \gamma \rightarrow \beta$ pathway for $\mathrm{Ti}$, and found the $\delta$ phase to be energetically unstable under hydrostatic conditions (Verma et al., 2007). The $\gamma$ phase was found to be elastically stable between $102 \mathrm{GPa}$ and $112 \mathrm{GPa}$. However, under non-hydrostatic conditions, the authors predicted the $\gamma$ phase to exist over a larger pressure range (Verma et al., 2007). The influence of anisotropic stresses under very non-hydrostatic conditions may support the existence of the $\delta$ phase reported in DAC experiments (Akahama et al., 2001; Vohra et al., 2001; Velisavljevic, n.d.).

The actual mechanism behind the martensitic transformation between the $\alpha, \beta$ and $\omega$ phases in Ti has been explored in a series of molecular dynamics simulations (Trinkle et al., 2003; Trinkle et al., 2005; Hennig et al., 2005; Hennig et al., 2008). The authors propose the lowest energy pathway for the $\alpha \rightarrow \omega$ transition to be the TAO-1, ("titanium alpha to omega"), in which atoms in the $\alpha$ phase transform through small shuffles and strains into the $\omega$ phase, without going through a metastable intermediate phase (Trinkle et al, 2003 \& Trinkle et al., 2005). The presence of impurities in Ti such as $\mathrm{O}, \mathrm{N}$ and $\mathrm{C}$ can affect the energy barrier to the $\omega$ phase and suppress the $\alpha \rightarrow \omega$ transformation (Hennig et al., 2005). Hennig et al. predicted the phase boundaries for Ti up to $15 \mathrm{GPa}$, and the $\alpha-\beta-\omega$ triple point at $8 \mathrm{GPa}$ and 1200 K. (Hennig et al., 2008).

Mei et al. studied the thermodynamic properties and phase diagram of $\mathrm{Ti}$ and predicted the $\mathrm{RP} \alpha \rightarrow \beta$ transition at $1114 \mathrm{~K}$ and the $\alpha-\omega-\beta$ triple point at $11.1 \mathrm{GPa}$ and $821 \mathrm{~K}$ (Mei et al., 2009). The $\alpha \rightarrow \omega$ transition was predicted at $1.8 \mathrm{GPa}$, slightly lower than experimental measurements. $\mathrm{Hu}$ et al. performed a detailed calculation to predict the phase diagram, thermal EOS and thermodynamic properties of Ti (Hu et al., 2010). The axial ratio of the $\alpha$ phase was predicted to be almost invariant with pressure, in agreement with the anvil study of Zhang et al., and with calculation (Zhang et al., 2008 \& Mei et al., 2009), but not with a DAC study in which the compression was found to be anisotropic (Errandonea et al., 2005). $\mathrm{Hu}$ et al. calculated the RT $\alpha \rightarrow \omega$ transition at $2.02 \mathrm{GPa}$ and the triple point at $9.78 \mathrm{GPa}$ and 
$931 \mathrm{~K}$, which is in close agreement with experiment (Bundy 1965 \& Zhang et al., 2008). The slope of the $\alpha-\omega$ boundary $(d T / d P=81 \mathrm{~K} / \mathrm{GPa})$ differs significantly to that measured by Zhang et al. in their cubic anvil study $(d T / d P=345 \mathrm{~K} / \mathrm{GPa})$ (Zhang et al., 2008). The slope of the $\omega-\beta$ boundary was calculated to be $2.4 \mathrm{~K} / \mathrm{GPa}$ (Hu et al., 2010), in good agreement with earlier measurements (Bundy, 1965). The predicted RT $\omega \rightarrow \gamma$ transformation at $110 \mathrm{GPa}$ concurred with the calculation of Verma et al. (Verma et al., 2007).

For reference, we show in figure 2 a representation of the Ti phase diagram as a function of pressure and temperature, based loosely on the experimental and theoretical studies discussed in this review.

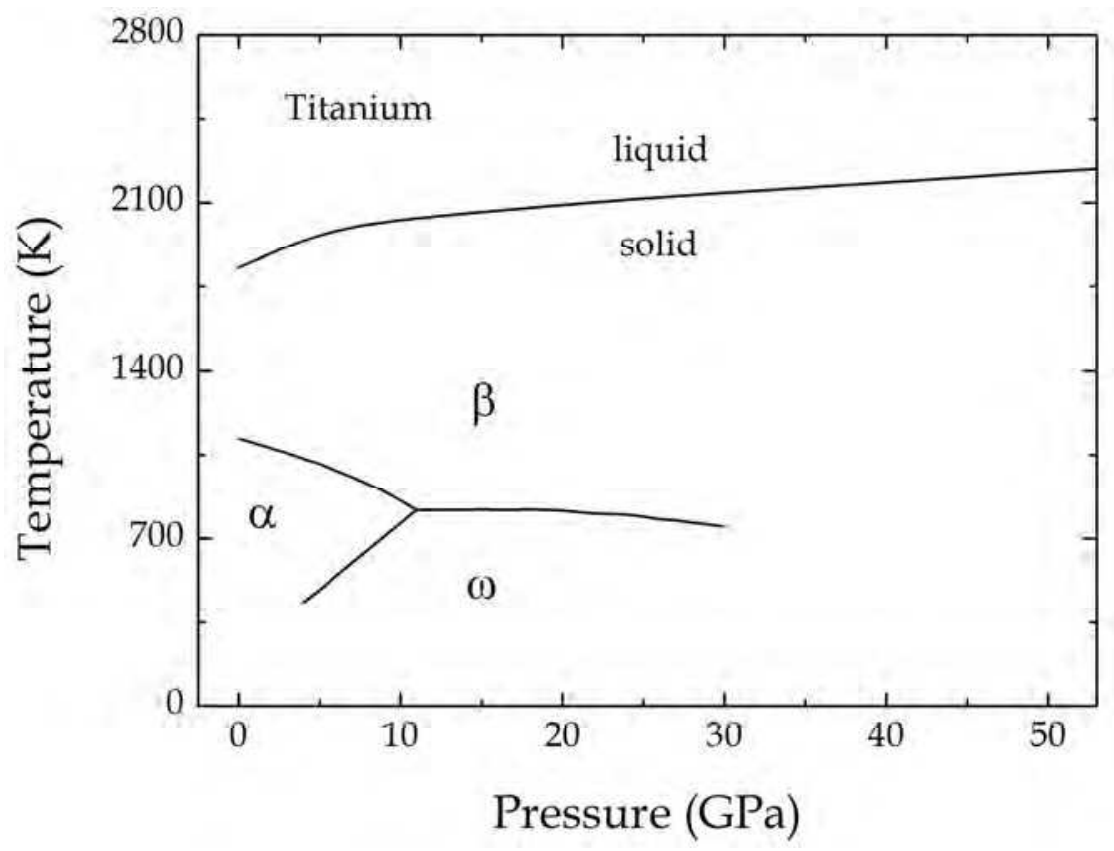

Fig. 2. The $P-T$ phase diagram of $\mathrm{Ti}$ (this is a representation based on published work).

\section{Ti alloys at high pressure}

Ti alloys are usually classified according to their phase stability. An $\alpha$ alloy consists mainly of an $\alpha$-phase stabilizing element such as $\mathrm{Al}, \mathrm{O}, \mathrm{N}$ or $\mathrm{C}$, which has the effect of extending the range of the more ductile $\alpha$-phase field to higher temperatures and higher pressures. Similarly, a $\beta$ alloy contains a $\beta$-phase stabilizing element such as $\mathrm{Mo}, \mathrm{V}$ or Ta, the presence of which will shift the $\beta$ phase field to lower temperatures. Ti alloys containing a combination of both $\alpha$-phase and $\beta$-phase stabilizing elements are by far the most widely used alloys commercially. At ambient conditions, the $\alpha+\beta$ alloys possess a $\beta$-phase fraction by volume that lies somewhere between 5 and $50 \%$ and so crystallizes predominantly in the $\alpha$ phase. Of particular importance is the $\alpha+\beta$ alloy Ti-6Al-4V (wt\%), which has found many commercial applications as a result of its superior material properties. The phase stability in 
$\mathrm{Ti}$ alloys is governed by the presence of impurities, and for a ternary alloy such as Ti-6Al$4 \mathrm{~V}$, the substitutional impurities, $\mathrm{Al}$ (which is an $\alpha$-phase stabilizer) and $\mathrm{V}$ (a $\beta$-phase stabilizer), influence the onset of the $\alpha \rightarrow \omega$ phase transformation by changing the $d$ electron concentration in the alloy (Vohra, 1979). The addition of $\mathrm{Al}$ reduces the $d$ band concentration, whilst the addition of $\mathrm{V}$ increases it by one, thus resulting in an overall reduction in the $d$ band concentration. Interstitial impurities such as $\mathrm{O}, \mathrm{N}$ and $\mathrm{C}$ can retard the $\alpha \rightarrow \omega$ transformation. Ab initio calculations have shown that the presence of these impurities can affect the relative phase stability and the energy barrier of the phase transformation (Hennig et al., 2005). The presence of impurities in the commercial Ti alloys $\mathrm{A}-70$ and $\mathrm{Ti}-6 \mathrm{Al}-4 \mathrm{~V}$, particularly $\mathrm{O}$ and $\mathrm{Al}$, suppresses the onset of $\alpha \rightarrow \omega$ phase transformation by increasing the energy and energy barrier of $\omega$ relative to $\alpha$ (Hennig et al., 2005). Thus, the stability range of the $\alpha$ phase at RT is increased. Hennig et al. predicted the RT $\alpha \rightarrow \omega$ phase transformations in A-70 and Ti-6Al-4V to occur at $31 \mathrm{GPa}$ and $63 \mathrm{GPa}$ respectively (Hennig et al., 2005).

\subsection{Ti-6Al-4V at high pressure}

As the most prevalent Ti alloy currently in commercial and industrial usage, it is perhaps not surprising that Ti-6Al-4V has received the most attention of all the Ti alloys at high pressure (Rosenberg \& Meybar, 1981; Gray et al., 1993; Cerreta et al., 2006; Chesnut et al., 2008; Halevy et al., 2010; Tegner et al., 2011; Tegner et al., n.d). The alloying of a metal can have substantial effects on its properties, which of course is the desired effect, and so it is important that we understand how the alloy responds to extremes of pressure.

\subsubsection{Dynamic compression of Ti-6Al-4V}

In the dynamic regime, the Hugoniot curve of Ti-6Al-4V was generated up to $14 \mathrm{GPa}$ using powder-gun driven shock waves and manganin stress gauges (Rosenberg \& Meybar, 1981). A break in the stress-particle velocity curve near $10 \mathrm{GPa}$ was indicative, the authors proposed, of a possible phase transformation, though they were unable to state unequivocally that the transformation was $\alpha \rightarrow \omega$ (Rosenberg \& Meybar, 1981). As part of a study to examine the effects of alloy chemistry on $\omega$ phase formation in Ti alloys, Gray et al., shocked Ti-6Al-4V (oxygen content 0.18 by weight \%) up to $25 \mathrm{GPa}$ and used VISAR to measure wave profiles (Gray et al., 1993). No evidence of a $\alpha \rightarrow \omega$ phase transformation was detected using VISAR, and using neutron diffraction to analyse the recovered specimen did not reveal the presence of $\omega$-phase structure.

\subsubsection{Static compression of Ti-6Al-4V}

The first study of Ti-6Al-4V in a DAC was reported by Chesnut et al., in which a sample was loaded into a 4:1 methanol: ethanol PTM and compressed to 37 GPa (Chesnut et al., 2008). The ambient conditions volume of Ti-6Al-4V (predominantly in the $\alpha$ phase) was measured using ADXD and found to be $V_{0}=17.208 \AA^{3} /$ atom. Chesnut et al. observed Ti-6Al-4V to undergo the $\alpha \rightarrow \omega$ phase transition at $\sim 27.3 \mathrm{GPa}$ (Chesnut et al., 2008). The $\omega$ phase was observed to be stable to $37 \mathrm{GPa}$ (the pressure limit of the experiment). The volume change across the phase boundary, at around 1\%, was considered too small to detect in shock experiments and may explain why this transformation has yet to be observed in 
dynamically driven Ti-6Al-4V (Rosenberg \& Meybar, 1981 \& Gray et al., 1993). Fitting a 3rd order Birch-Murnaghan EOS (Birch 1952) to the data generated an isothermal bulk modulus (which is a measure of the incompressibility of a material) for the $\alpha$ phase of $K=125.24 \mathrm{GPa}$ and the pressure derivative of the isothermal bulk modulus, $K^{\prime}=2.409$.

Halevy et al. compressed a sample of Ti-6Al-4V to $32.4 \mathrm{GPa}$ in a DAC, and using energy dispersive X-ray diffraction (EDXD), did not observe a transformation to the $\omega$ phase (Halevy et al., 2010). A Vinet EOS (Vinet et al., 1987) fit to the experimental data returned values for the isothermal bulk modulus and the pressure derivative of $K=154 \pm 11 \mathrm{GPa}$ and $K^{\prime}=5.4 \pm 1.4$. No mention was made of a PTM being used in this study.

\subsubsection{Effects of pressure media on the phase relations of Ti-6Al-4V}

In the most recent DAC study of Ti-6Al-4V, conducted by two of us (MacLeod and Cynn), powdered polycrystalline samples were embedded in a variety of PTMs to investigate the effects of the pressure environment on the RT $\alpha \rightarrow \omega$ phase transformation (Tegner et al., 2011; Tegner et al., n.d.). ADXD data were collected at the High Pressure Collaborative Access Team (HP-CAT) beamline 16-IDB at the Advanced Photon Source (APS) in Chicago, for Ti-6Al-4V samples embedded in neon, 4:1 methanol: ethanol and mineral oil. The oxygen content of the Ti-6Al-4V was 0.123 by weight $\%$. The ambient conditions volume in the $\alpha$ phase was measured to be $V_{0}=17.252 \AA^{3} /$ atom. We observed the $\alpha \rightarrow \omega$ phase transformation to occur at $32.7 \mathrm{GPa}$ for Ti-6Al-4V in the neon PTM, 31.2 GPa for the methanol: ethanol PTM and 26.2 GPa for the mineral oil PTM (in order of decreasing hydrostaticity in the PTM). At elevated pressures, ultimately all PTMs will become nonhydrostatic in nature (see Klotz et al., 2009, for a general discussion on the hydrostatic limits of various pressure media) and so it becomes more difficult to quantify the dependence of the $\alpha \rightarrow \omega$ phase transformation pressure in Ti-6Al-4V based on the hydrostaticity of the pressure environment, unlike in $\mathrm{Ti}$ where the transition is observed at a much lower pressure (see Errandonea et al., 2005).

A coexistence of the $\alpha$ and $\omega$ phases was observed over a largish pressure range (of the order $10 \mathrm{GPa}$ or greater) for the different PTMs, similar to what was observed for Ti. A stacked plot of ADXD patterns, showing the structural response of Ti-6Al-4V to applied pressure, in a neon PTM, is shown in figure 3. Reflections from the Ti-6Al-4V sample in both $\alpha$ and $\omega$ phases are present, together with reflections from neon (PTM) and copper (the pressure marker).

In figure 3, at $30.7 \mathrm{GPa}$, we observe the (100), (002), (101), (102) and (110) peaks that are characteristic of the $\alpha$ phase. The dominant (110/101) reflections corresponding to the $\omega$ phase appear at $\sim 32.7 \mathrm{GPa}$ (between the $\alpha$ phase (002) and (101) peaks) and then gradually grow in magnitude with pressure, whilst simultaneously the $\alpha$ phase peaks diminish in intensity, until the pressure reaches $\sim 44 \mathrm{GPa}$. By $44 \mathrm{GPa}$, the $\alpha \rightarrow \omega$ transformation is virtually completed. The $\omega$ phase (001), (201) and (210) reflections appear at a slightly higher pressure than the $(110 / 101)$ peaks, at around $36 \mathrm{GPa}$ to $39 \mathrm{GPa}$. In all, up to $10 \omega$ phase peaks were indexed in this study. It is clear from figure 3 that both the $\alpha$ and $\omega$ phases coexist over a large pressure range, of the order of $10 \mathrm{GPa}$ (between $\sim 32.7 \mathrm{GPa}$ and $\sim 44$ GPa). We observed similar behaviour for Ti-6Al-4V embedded in methanol: ethanol and mineral oil, and also for an experiment with no PTM (Tegner et al., 2011). For Ti-6Al-4V embedded in methanol: ethanol, we decompressed our DAC from $75 \mathrm{GPa}$ to ambient and observed the $\omega$ phase to gradually revert back to the $\alpha$ phase. 


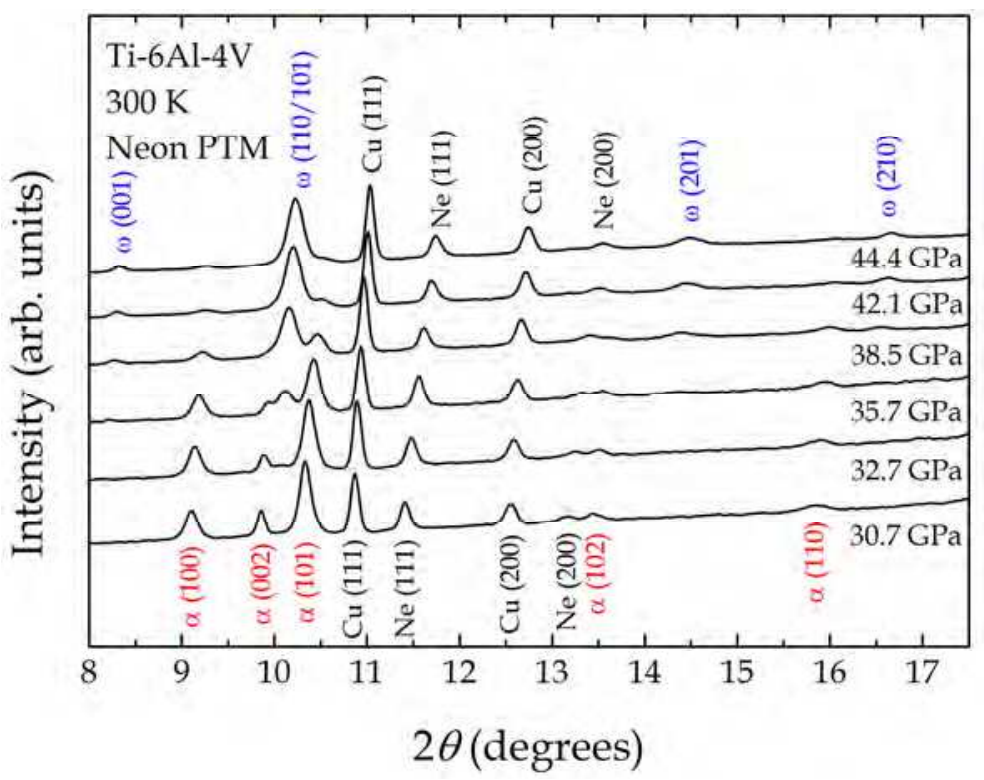

Fig. 3. A stack of ADXD patterns showing structural change in Ti-6Al-4V with increasing pressure (Tegnet et al., 2011).

We determined the pressure in our experiments by analysing the reflections from the pressure markers (either Ta or $\mathrm{Cu}$ ) and using a known EOS from previous shock measurements. In the case of the $\mathrm{Cu}$ marker used in the compression of Ti-6Al-4V in a neon PTM (figure 3), we used a well known Cu shock study (Carter et al., 1971).

We now show in the $P-V$ plot in figure 4 our measurements for Ti-6Al-4V embedded in a methanol: ethanol PTM, alongside previous DAC measurements (Chesnut et al., 2008 \& Halevy et al., 2010). There is good agreement between the Chesnut et al. and our (Tegner et al., n.d.) measurements (both using a methanol: ethanol PTM), but not with the Halevy et al measurements (there was no mention of a PTM being used in their study). We measured the $P_{\alpha \rightarrow \omega}=31.2 \mathrm{GPa}$, and an isothermal bulk modulus of $K=115 \pm 3 \mathrm{GPa}$ and pressure derivative of $K^{\prime}=3.22 \pm 0.22$ after fitting a Vinet EOS (Vinet et al., 1987) to the data (Tegner et al., 2011). The volume change across the $\alpha-\omega$ phase boundary was measured to be less than $1 \%$, in agreement with the previous methanol: ethanol study (Chesnut et al., 2008).

The axial ratio $(c / a)$ for the $\alpha$ phase of Ti-6Al-4V (at ambient conditions) was measured to be 1.602, which is slightly higher than that reported for pure Ti (1.587), but is an expected result due to the presence of the $\alpha$ stabiliser $\mathrm{Al}$ (possessing a smaller atomic radius than $\mathrm{Ti}$ ) in the alloy.

For the $\alpha$ phase of Ti-6Al-4V, in a methanol: ethanol PTM, we found the axial ratio to be almost constant between 1.600 and 1.602 up to $42 \mathrm{GPa}$, see figure 5 . The measured $c / a$ ratios for the $\omega$ phase are also effectively constant between $34 \mathrm{GPa}$ and $74 \mathrm{GPa}$, varying between 0.616 and 0.617 . We find similar results for a loading of Ti-6Al-4V in a neon PTM. These measurements are in broad agreement with those reported by Errandonea et al. for pure Ti 


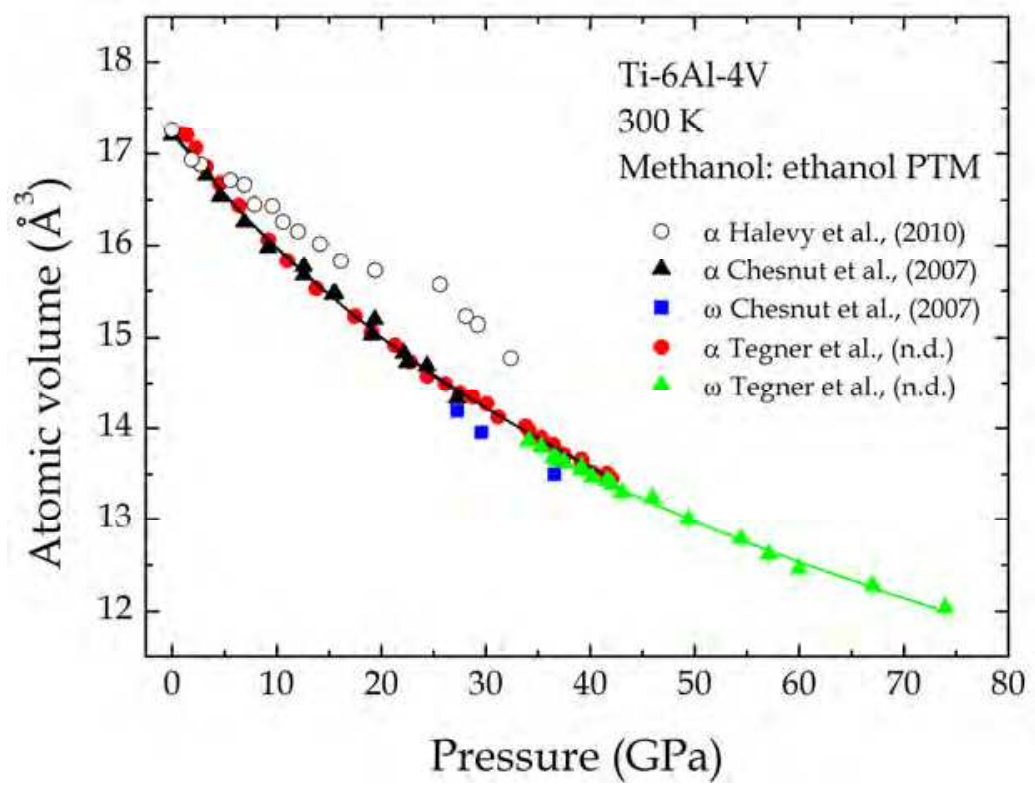

Fig. 4. The $P-V$ plot for Ti-6Al-4V in a methanol: ethanol PTM (Chesnut et al., 2008 \& Tegner et al., 2011) and also for an unspecified PTM (Halevy et al., 2010).

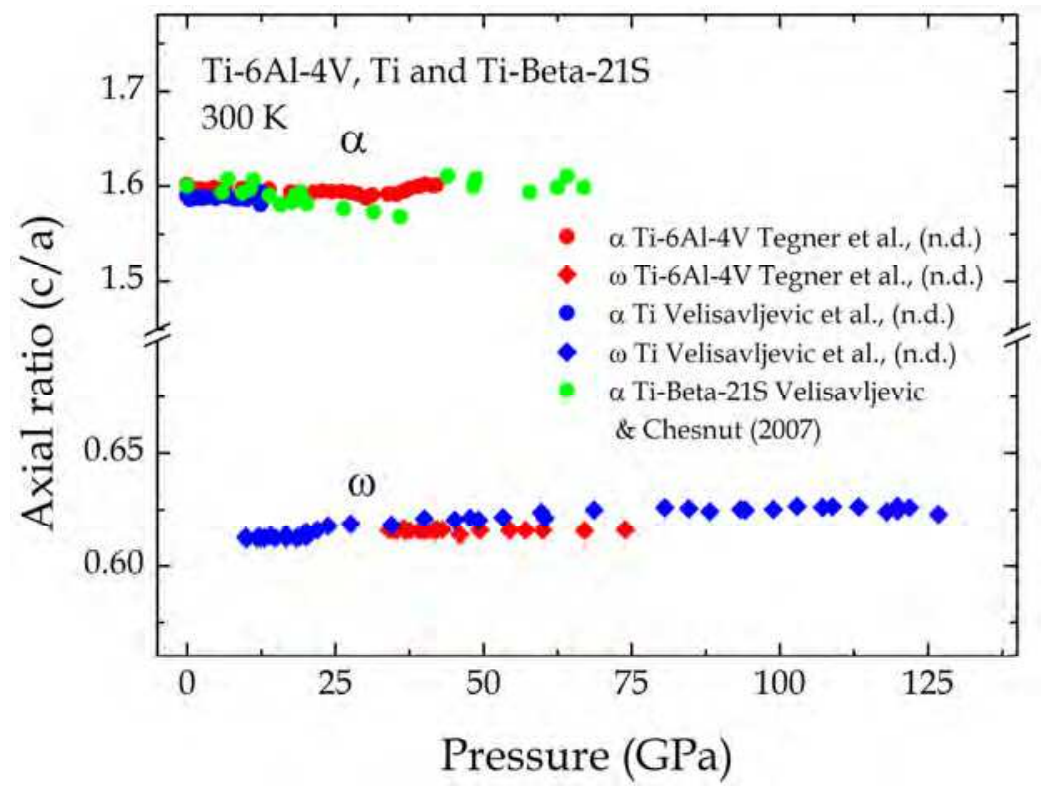

Fig. 5. The axial ratios $(c / a)$ for the $\alpha$ and $\omega$ phases of Ti-6Al-4V in a methanol: ethanol PTM (Tegner et al., n.d.), Ti with no PTM (Velisavljevic et al., n.d.) and Ti-Beta-21S in a methanol: ethanol PTM (Velisavljevic \& Chesnut, 2007). 
(Errandonea et al., 2005). We also include for reference in figure 5 our axial ratio results for nano-Ti with no PTM (Velisavljevic et al., n.d.) and Ti-Beta-21S in a methanol: ethanol PTM (Velisavljevic \& Chesnut, 2007) (see section 3.2). The $c / a$ ratio for nano-Ti in the $\omega$ phase had a steady value of 0.612 initially, and as the pressure was increased above $20 \mathrm{GPa}$, this value increased slightly to 0.626 and then levelled off above $80 \mathrm{GPa}$. Under compression, the $\omega$ phase of Ti-6Al-4V in a methanol: ethanol PTM was observed to be stable to $115 \mathrm{GPa}$ (the pressure limit in this experiment).

We observed, in further RT volume compression experiments of Ti-6Al-4V embedded in neon and mineral oil PTMs, a gradual transformation from the $\omega$ phase to the body-centredcubic $\beta$ phase (space group Im3m) (Tegner et al., 2010 \& Tegner et al., n.d.). For both neon and mineral oil PTMs, the transformation is completed between $115 \mathrm{GPa}$ and $125 \mathrm{GPa}$. The $\beta$ phase is formed by the splitting of the alternating (001) plane along the $c$ axis of the $\omega$ phase into two (111) planes of the $\beta$ phase (Xia et al., 1990a). All the $\beta$ phase peaks are therefore contained in the $\omega$ diffraction pattern (that is, the peaks are coincident). With no detectable volume change from $\omega \rightarrow \beta$, it was not possible to ascertain over what pressure range both phases coexisted. The disappearance of the $\omega$ phase (001), (210) and (002) peaks was the only indication that a solid-solid phase transformation had occurred. We observed the $\beta$ phase of Ti-6Al-4V to be stable to at least $221 \mathrm{GPa}$ (the pressure limit of the experiment) in a mineral oil PTM (Tegner et al., 2011) and stable to $130 \mathrm{GPa}$ (the pressure limit of the experiment) in the neon PTM (Tegner et al., n.d.).

The $P-V$ plot for Ti-6Al-4V in a mineral oil PTM is shown in figure 6. No intermediate phases were observed in this experiment. For comparison, we include the $P-V$ data for nanoTi (Velisavljevic, n.d.), Ti-6Al-4V (Chesnut \& Velisavljevic, 2008) and (see section 3) Ti-Beta$21 S$ (Velisavljevic \& Chesnut, 2007). Verma et al. proposed that the observations of the $\delta$ phase in Ti DAC experiments were likely caused by the presence of non-hydrostatic stresses and that the transition sequence $\omega \rightarrow \gamma \rightarrow \beta$ is thermodynamically preferable, with the intermediate $\gamma$ phase existing over a range of at least $10 \mathrm{GPa}$ (Verma et al., 2007). In our Ti$6 \mathrm{Al}-4 \mathrm{~V}$ study, we collected ADXD patterns with pressure steps $\sim 5 \mathrm{GPa}$ and found no evidence for an intermediate $\gamma$ phase in Ti-6Al-4V. Nor was there any evidence for an orthorhombic $\delta$ phase. The Ti-6Al-4V sample was embedded in a PTM (albeit the very nonhydrostatic mineral oil and neon PTMs above $100 \mathrm{GPa}$ ). Our own calculations predict the transformation pathway for Ti-6Al-4V to be $\alpha \rightarrow \omega \rightarrow \beta$, with $\alpha \rightarrow \omega$ occurring at $24 \mathrm{GPa}$ and $\omega \rightarrow \beta$ at $\sim 105 \mathrm{GPa}$, in agreement with experiment (Tegner et al., 2010). Above $110 \mathrm{GPa}$ we find that the orthorhombic $\gamma$ and $\delta$ phases relax to the cubic $\beta$ phase under hydrostatic conditions. As far as we know, all Ti DAC experiments compressed above $100 \mathrm{GPa}$ have had samples compressed in the absence of a PTM (Vohra et al., 2001; Akahama et al., 2001; Velisavljevic et al., n.d.).

In figure 7, an integrated ADXD pattern is shown of Ti-6Al-4V in a neon PTM, collected at 129 GPa (Tegner et al., n.d.). The peaks are indexed as the $\beta$-phase reflections (110), (200), (211) and (220) and face-centred cubic (fcc) neon (111), (002) and (220). The pressures were calculated using a previous static high pressure EOS study of neon (Dewaele et al., 2008). We performed a Rietveld analysis of our diffraction patterns to confirm the crystal structure of Ti-6Al-4V to be the $\beta$ phase. 


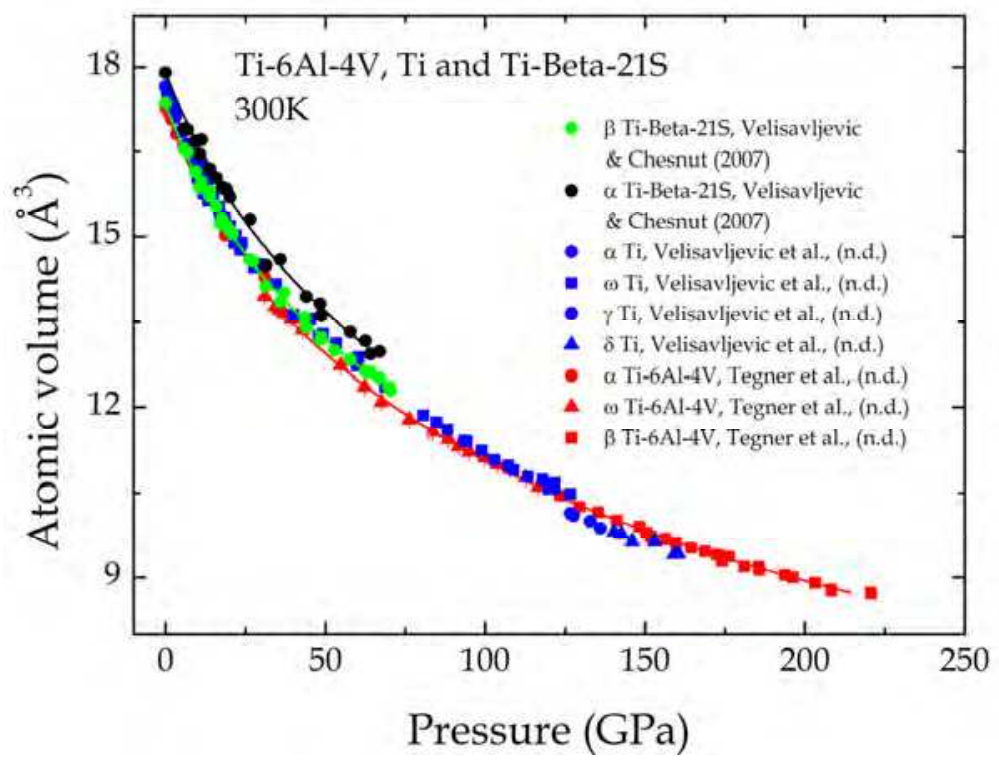

Fig. 6. The $P-V$ plot for Ti-6Al-4V in a mineral oil PTM (Tegner et al., n.d.), Ti with no PTM (Velisavljevic et al., n.d.) and Ti-Beta-21S in a methanol: ethanol PTM (Velisavljevic \& Chesnut, 2007).

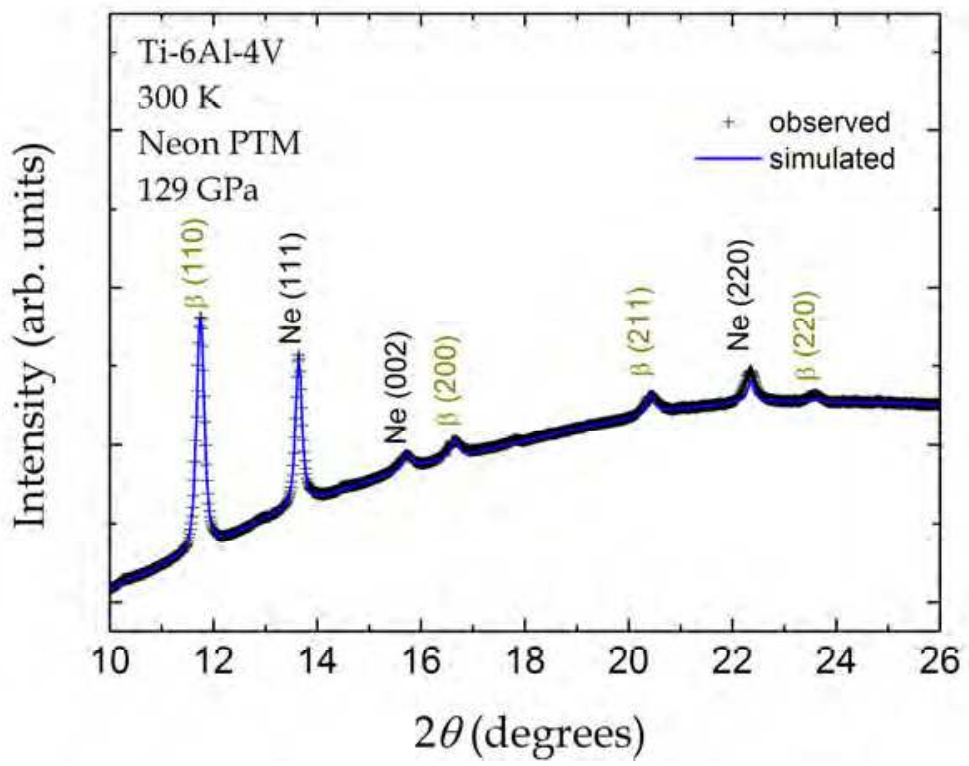

Fig. 7. An integrated ADXD pattern and Rietveld analysis for $\beta$ (bcc) phase Ti-6Al-4V at 129 GPa and embedded in a neon PTM (Tegner et al., n.d.). The neon was used as both a PTM and pressure marker. 
The observation of the $\alpha \rightarrow \omega \rightarrow \beta$ transformation pathway at RT for Ti-6Al-4V suggests that the slope of the $\omega-\beta$ phase boundary (see boundary in figure 2 ) is negative at high pressures, or that there are two separate areas of $\beta$ phase separated by the $\omega$ phase (see for example, Xia, 1990a).

\subsection{Absence of the $\omega$ phase in the Ti-beta-21S alloy at high pressure}

In cases where a sufficient amount of alloying elements are introduced, known as Mo equivalent $\left(\mathrm{Mo}_{\mathrm{eq}}\right)$, alloys with up to $50 \% \beta$ phase can be recovered after temperature treatment (Bania, 1993). The large $\beta$ phase concentration can have a significant effect on the pseudo-elastic response (Zhou et al., 2004), while also effecting structural phase stability at high pressures. One example is the Mo rich Ti-Beta-21S (also known as TIMETAL21S®) alloy, which is a $\beta$-stabilized alloy with $15 \mathrm{wt} . \% \mathrm{Mo}, 3 \mathrm{wt} . \% \mathrm{Al}, 2.7 \mathrm{wt} . \% \mathrm{Nb}, 0.2 \mathrm{wt} \% \mathrm{Si}$, and the remainder made up by Ti. The standard "solution-treated-and-aged" (STA) heat treatment, in which the samples were heated to $1098 \mathrm{~K}$, held for 30 minutes, cooled to RT (air-cooled equivalent rate), subsequently heated to $828 \mathrm{~K}$, held for 8 hours, and again cooled to RT, produced a sample resulting in a mixture of $\alpha+\beta$ phases, with $29 \%-42 \%$ in the $\alpha$ phase (Velisavljevic \& Chesnut, 2007; Honnell et al., 2007).

An initial ADXD pattern collected at ambient conditions clearly shows the sample is composed of both the $\alpha$ and $\beta$ phases (Velisavljevic \& Chesnut, 2007), as shown in figure 8. A close examination of the diffraction pattern does not indicate the existence of any peaks that could not be attributed to either the $\alpha$ or $\beta$ phase, and in particular, there is no evidence of $\omega$ phase in the sample. Cold compression of the sample in a methanol: ethanol PTM, in a DAC, shows very little change at low pressure. Up to $\sim 11 \mathrm{GPa}$ both $\alpha$ and $\beta$ phases appear to be stable, including the axial ratio of the $\alpha$ phase, which remains fairly constant near the initial value of $c_{0} / a_{0}=1.601$. Comparison of the axial ratio of the $\alpha$ phase of Ti-Beta-21S and the $c_{0} / a_{0}$ value of 1.602 reported for the $\alpha$ phase of Ti-6Al-4V indicates that the inclusion of $\mathrm{Mo}$, which has a larger atomic radius than $\mathrm{Ti}$, does not have a significant effect on the initial axial ratio. However, the measured volume $V_{0}=17.912 \AA^{3} /$ atom $\left(a_{0}=2.956 \pm 0.002 \AA\right.$ and $c_{0}$ $=4.734 \pm 0.025 \AA$ ) for the $\alpha$ phase of Ti-Beta-21S is larger than both the $V_{0}=17.355 \AA^{3} /$ atom $\left(a_{0}=3.262 \pm 0.004 \AA\right)$ measured for the $\beta$ phase of Ti-Beta-21S and values reported for Ti$6 \mathrm{Al}-4 \mathrm{~V}$, which would suggest that a significant amount of alloying elements are still present in the $\alpha$ phase portions of the sample. Above $11 \mathrm{GPa}$, anisotropic compression of the $\alpha$ phase is observed, which leads to a steady decrease in the $c / a$ ratio down to 1.568 at $36 \mathrm{GPa}$, as shown in figure 5. A sudden increase in the axial ratio up to 1.613 at $44 \mathrm{GPa}$, followed by a steady value up to $67 \mathrm{GPa}$ is then observed. Over this same pressure range, besides a steady decrease in volume, no significant changes are observed for the $\beta$ phase. The sudden change in the axial ratio in the $36-44 \mathrm{GPa}$ region could denote a potential first order isostructural phase transition. Over the same pressure region of $36-44 \mathrm{GPa}$, no evidence of appearance of the $\omega$ phase or any other new phases could be detected.

With pressure increased to $58 \mathrm{GPa}$, the sample remains stable, as a mixture of $\alpha+\beta$ phases. However, above this pressure a relative change in the measured peak intensities between the two phases indicates the onset of a structural phase transition (Velisavljevic \& Chesnut, 2007). A comparison of the intensity change of the (102) $\alpha$-phase peak and the 
(200) $\beta$-phase peak indicates a steady disappearance of the $\alpha$ phase and a transition of the sample to $100 \% \beta$ phase above $67 \mathrm{GPa}$. The ADXD patterns collected at $18 \mathrm{GPa}$ and 48 GPa in figure 8 show the mixture of the $\alpha+\beta$ phases. At $71 \mathrm{GPa}$ in figure 8 , only $\beta$ phase peaks are now present. From the experimental data, a Vinet EOS fit returned values of $K=$ $119 \mathrm{GPa}$ and $K^{\prime}=3.4$ for the $\alpha$ phase and $K=109 \mathrm{GPa}$ and $K^{\prime}=3.8$ for the $\beta$ phase (Honnell et al., 2007), and for a Birch-Murnaghan EOS fit, values of $K=117 \mathrm{GPa}$ and $K^{\prime}=$ 3.4 for the $\alpha$ phase and $K=110 \mathrm{GPa}$ and $K^{\prime}=3.7$ for the $\beta$ phase (Velisavljevic \& Chesnut, 2007) were obtained. Although, as previously mentioned, there is a volume difference observed between the $\alpha$ phase of Ti-Beta-21S and Ti-6Al-4V, the overall compressibility and EOS of Ti-Beta-21S are in good agreement with the various EOS values generated for Ti-6Al-4V, as shown in figure 6.

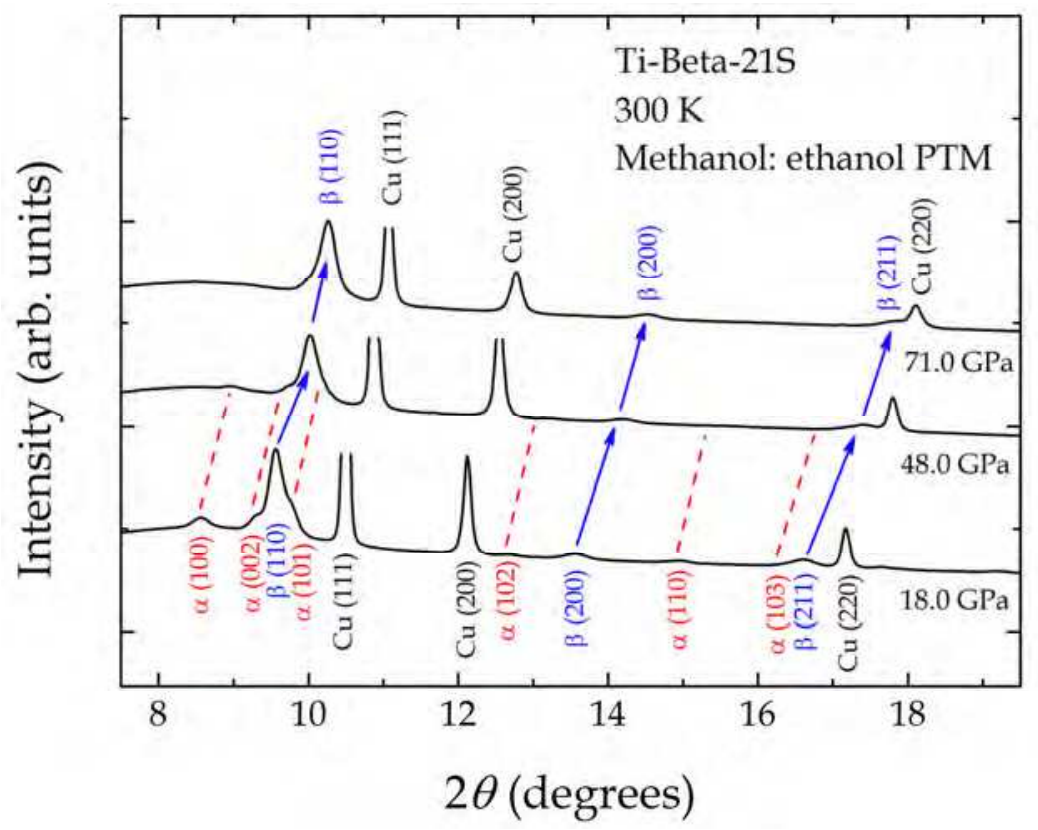

Fig. 8. A stack of ADXD patterns showing structural change in Ti-Beta-21S with increasing pressure. Initially sample is composed of mixture $\alpha+\beta$ phase and with pressure increase sample transforms completely to $\beta$ phase $-\omega$ or other intermediate phases were not observed at any point. Additional peaks in ADXD spectra belong to $\mathrm{Cu}$, which was used as a pressure marker.

For data collection, and the values reported here for Ti-6Al-4V and Ti-beta-21S, synchrotron source monochromatic X-ray beams were used. The image plate detectors available at the synchrotrons had pixel sizes of $100 \mu \mathrm{m}^{2}$ and so the diffraction patterns were generated with a resolution $\sim \Delta \mathrm{d} / \mathrm{d}=10^{-3}$. As a consequence, the uncertainties in the measured volume data were of the order of $\sim 0.3 \%$. This is consistent with the different values reported for the volume of Ti-6Al-4V at ambient conditions by the various authors. For example, Tegner et al. reported $V_{0}=17.252 \AA^{3} /$ atom (Tegner et al., 2010) whereas 
Chesnut \& Velisavljevic measured a $0.25 \%$ smaller value, $\mathrm{V}_{0}=17.208 \AA^{3} /$ atom (Chesnut \& Velisavljevic, 2007).

\section{Discussion}

A P-T phase diagram for Ti (Errandonea et al., 2001; Errandonea et al., 2005; Pecker et al., 2005; Zhang et al., 2008; Mei et al, 2009; Hu et al., 2010) and Ti-6Al-4V (Chesnut et al., 2008, Tegner et al., 2010) summarising the current state of knowledge of the phase relations of these systems up to $125 \mathrm{GPa}$ is shown in figure 9. There is good agreement between experiment and theory for the location of the Ti $\alpha-\omega$ phase boundary, and also the melt curve, but the location and slope of the $\omega-\beta$ boundary is still in dispute and requires more study for clarification. Phase stability and the effects of anisotropic stresses on the $\alpha \rightarrow \omega$ transition in $\mathrm{Ti}$ is an issue that also requires more attention at high temperature. Very little is known about $\mathrm{Ti}$ alloys at high pressure, and even less at high pressure and high temperature. In figure 9, we suggest possible phase boundaries for Ti-6Al-4V as a dashed blue line. We are unsure about the exact location, or slope even, of the $\alpha-\omega$ and $\alpha-\beta$ boundary for Ti-6Al-4V at high temperature.

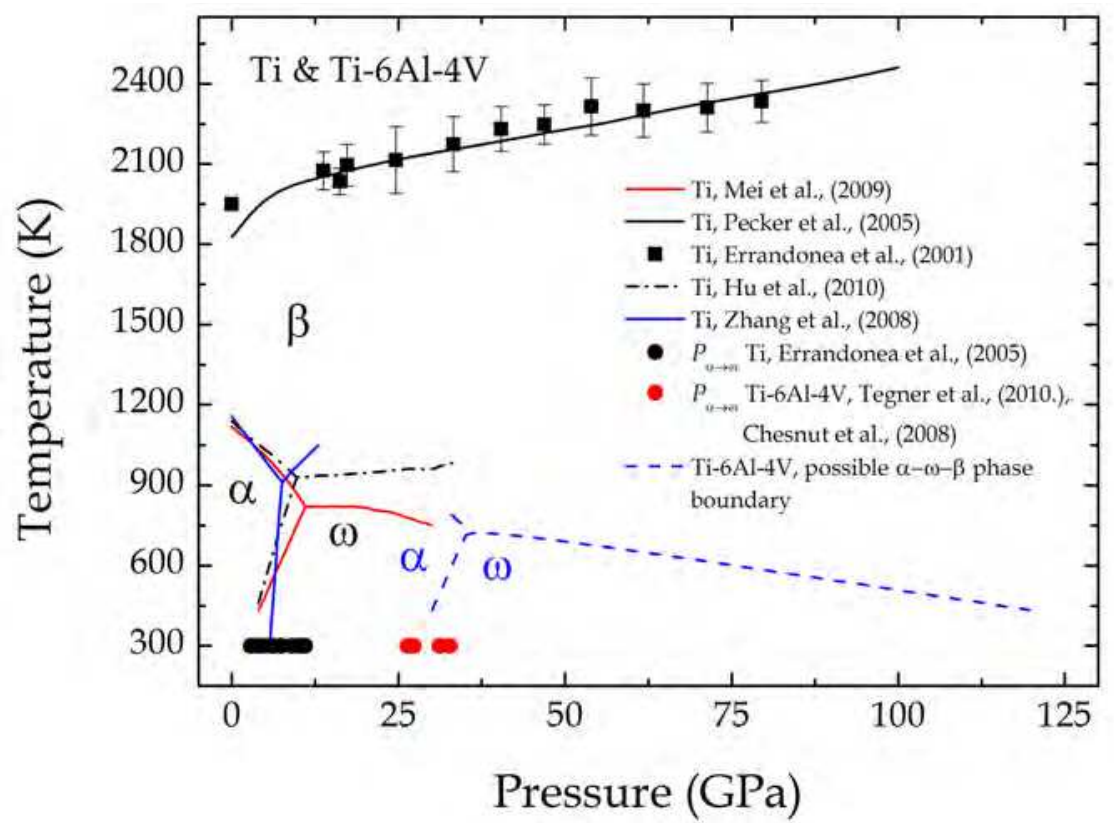

Fig. 9. The combined P-T phase diagram of Ti (Errandonea et al., 2001; Errandonea et al., 2005; Pecker et al., 2005; Zhang et al., 2008; Mei et al, 2009; Hu et al., 2010) and Ti-6Al-4V (Chesnut et al., 2008, Tegner et al., 2010). The possible phase boundaries for the $\alpha+\beta$ alloy Ti$6 \mathrm{Al}-4 \mathrm{~V}(\mathrm{wt} \%)$ are suggested by us as the dashed blue line. 
By comparing our high pressure Ti-Beta-21S (Velisavljevic \& Chesnut, 2007), Ti-6Al-4V (Chesnut et al., 2008; Tegner et al., 2010; Tegner et al., n.d.) and nano-Ti (Velisavljevic et al., n.d.) data, we observe that the addition of alloying elements can have a significant influence on the structural phase transition sequence in these metals at high pressure and temperature. For example, the presence of alloys and interstitial impurities in Ti-6Al-4V suppresses the onset of the $\alpha \rightarrow \omega$ phase transformation, thus ensuring the predominance of the $\alpha$-phase alloy over a much larger pressure range than exists for pure $\mathrm{Ti}$, which is desirable in industrial and commercial applications. The main effect observed in Ti-Beta-21S is the complete suppression of the brittle $\omega$ phase at high pressures.

However, it is also important to recognize that changes in the electronic configuration cause changes in crystal structures. These changes can be induced either by an increase in pressure or an increase in the occupancy of the $d$ bands. The structural trend exhibited by the $3 d, 4 d$, and $5 d$ transition metals is well known. The variation in the electronic configuration affects crystal structures and mechanical properties such as microstructures and dislocations. For industrial applications, it is the machineability and superior mechanical properties that are of paramount interest. Pressure allows us to measure the differences in crystal structures induced by changes in the electronic configuration.

Among the Group IV elements, similar structural changes occur. Group IV elements and their alloys apparently favor a transformation pathway $\alpha \rightarrow \omega \rightarrow \beta$ at high pressure, based on recent DAC experiments and theoretical calculations. The intermediate structures, $\delta$ and $\gamma$, appear to be metastable and are shear driven. Based on the experimental results one can conclude that high pressure structural phase transitions in $\mathrm{Ti}$ and $\mathrm{Ti}$ alloys are highly susceptible to loading conditions and stress distribution, as shown from experiments using various PMTs. However, stability of various phases can be controlled by other variables, such as alloying, which can change electronic structure by increasing/decreasing $d$ band occupancy, inclusion of interstitial impurities, which help reduce shear deformation, and in some cases it appears that shear driven structural phase transitions can be controlled by varying sample grain size as well. Although these factors play a significant role in controlling structural phase transitions they appear to have only a slight effect on the initial compressibility (i.e. EOS) - measured $\alpha$ phase EOS parameters for Ti-Beta-21S, Ti-6Al-4V, Ti, and nano-Ti are all relatively close with values of $K=115$ $125 \mathrm{GPa}$ and $K^{\prime}=2.4-3.9$. Overall it appears that with stress conditions, grain size, and presence of impurities, there is a systematic shift of the transition pressure $P_{\alpha \rightarrow \omega}$, as the transition pressure increases with improved hydrostaticity of the pressure environment and by grain size reduction.

\section{Acknowledgements}

SM would like to acknowledge the support of Professor Malcolm McMahon and Dr John Proctor of the Centre for Science at Extreme Conditions (CSEC), Edinburgh University, in collecting the Ti-6Al-4V data. This work was performed under the auspices of the U.S. Department of Energy by Lawrence Livermore National Laboratory in part under contract W-7405-Eng-48 and in part under Contract DE-AC52-07NA27344. LANL is operated by 
LANS, LLC for the DOE-NNSA - this work was, in part, supported by the US DOE under contract \# DE-AC52-06NA25396. HP-CAT is supported by CIW, CDAC, UNLV and LLNL through funding from DOE-NNSA, DOE-BES and NSF. APS is supported by DOE-BES under Contract No. DE-AC02-06CH11357.

\section{References}

Ahuja, R.; Dubrovinsky, L.; Dubrovinskaia, N.; Osorio Guillen, J.M.; Mattessini, M.; Johansson, B. and Le Bihan T. (2004). Titanium metal at high pressure: Synchrotron experiments and $a b$ initio calculations. Physical Review B, Vol.69, pp. 184102-1184102-4

Akahama, Y.; Kawamura, H. \& Le Bihan T. (2001). New $\delta$ (Distorted-bcc) Titanium to 220 GPa. Physical Review Letters, Vol.87, No.27, pp. 2755031-2755034

Bania, B.J.. (1993). Beta Titanium alloys and their role in the Titanium industry. In: Eylon D, Boyer R, Koss D, Editors, Beta Titanium alloys in the 1990's TMS, ISBN 0-87339-200-0, Warrendale, USA, February 1993

Birch, F. (1952). Elasticity and constitution of the Earth's interior. Journal of Geophysical Research, Vol.57, pp. 227-286

Bundy, F.P. (1963). General Electric Report No. 63-RL-3481C (unpublished)

Bundy, F.P. (1965). Formation of New Materials and Structures by High-Pressure Treatment. Irreversible Effects of High Pressure and Temperature on Materials, ASTM Special Technical Publication No. 374, Philadelphia, February, 1964

Carter, W.J.; Marsh, S.P.; Fritz, J.N. \& McQueen, R.G. (1971). The Equation of State of Selected Materials for High-Pressure References. National Bureau of Standards Special Publication 326: Accurate Characterisation of the High-Pressure Environment, pp. 147158, Gaithersburg, Md., October 14-18, 1968

Cerreta, E.; Gray III, G.T.; Lawson, A.C.; Mason, T.A. \& Morris, C.E. (2006). The influence of oxygen content on the $\alpha$ to $\omega$ phase transformation and shock hardening of titanium. Journal of Applied Physics, Vol.100, 013530-1-013530-9

Chesnut, G.N.; Velisavljevic, N. \& Sanchez, L. (2008). Static High pressure X-ray Diffraction of Ti-6Al-4V. Proceedings of the American Physical Society Topical Group on Shock Compression of Condensed Matter - 2007, pp. 27-30, ISBN 978-0735404694, Kohala Coast, Hawaii, USA, June 24-29, 2007

Dewaele, A.; Datchi, F.; Loubeyre, P. \& Mezouar, M. (2008). High pressure-high temperature equations of state of neon and diamond. Physical Review B, Vol.77, pp. 094106-1094106-9

Errandonea, D.; Schwager, B.; Ditz, R.; Gessmann, C.; Boehler, R. \& Ross, M. (2001). Systematics of transition-metal melting. Physical Review B, Vol.63, pp. 132104-1132104-4

Errandonea, D.; Meng, Y.; Somayazulu, M. \& Häusermann, D. (2005). Pressure-induced a $\rightarrow$ $\omega$ transition in titanium metal: a systematic study of the effects of uniaxial stress. Physica B, Vol.355, pp. 116-125 
Gray, G.T.; Morris, C.E. \& Lawson, A.C. (1993). Omega phase formation in Titanium and Titanium alloys. Proceedings of Titanium '92: Science and Technology, ISBN 0873392221, San Diego, USA, June 1992

Greeff, C.W.; Trinkle, D.R. \& Albers, R.C. (2001). Shock-induced a- $\omega$ transition in titanium. Journal of Applied Physics, Vol.90, No.5, pp. 2221-2226

Gupta, S.C.; Joshi, K.D. \& Banerjee, S. (2008). Experimental and Theoretical Investigations on $d$ and $f$ electron Systems under High Pressure. Metallurgical and Materials Transactions A, Vol.39A, pp. 1593-1601

Halevy, I.; Zamir, G.; Winterrose, M.; Sanjit, G.; Grandini, C.R. \& Moreno-Gobbi, A. (2010). Crystallographic structure of Ti-6Al-4V, Ti-HP and Ti-CP under High-Pressure. Journal of Physics: Conference Series, Vol.215, pp. 1-9

Hennig, R.G.; Trinkle, D.R.; Bouchet, J.; Srinivasan, S.G.; Albers, R.C. \& Wilkins, J.W. (2005). Impurities block the $\alpha$ to $\omega$ martensitic transformation in titanium. Nature, Vol.4, pp. 129-133

Hennig, R.G.; Lenosky, T.J.; Trinkle, D.R.; Rudin, S.P. \& Wilkins, J.W. (2008). Classical potential describes martensitic phase transformations between the $\alpha, \beta$, and $\omega$ titanium phases. Physical Review B, Vol.78, pp. 054121-1-054121-10

Honnell, K.G.; Velisavljevic, N.; Adams, C.D.; Rigg, P.A.; Chesnut, G.N.; Aikin Jr, R.M \& Boettger, J.C. (2007). Equation of State for Ti-beta-21S. Compression of Condensed Matter - 2007, edited by M. Elert, M.D. Furnish, R. Chau, N.C. Holmes and J. Nguyen, Conference Proceedings of the APS topical group on SCCM, (AIP, New York), 2007, Pt. 1, p.55.

Hu, C.-E.; Zeng, Z.-Y.; Zhang, L.; Chen, X.-R.; Cai, L.-C. \& Alfè, D. (2010). Theoretical investigation of the high pressure structure, lattice dynamics, phase transition, and thermal equation of state of titanium metal. Journal of Applied Physics, Vol.107, pp. 093509-1-093509-10

Jamieson, J.C. (1963). Crystal structure of Titanium, Zirconium, and Hafnium at high pressures. Science Vol.140, pp. 72-73

Jayaraman, A.; Klement, W. \& Kennedy G.C. (1963). Solid-Solid Transitions in Titanium and Zirconium at High Pressures. Physical Review, Vol.131, No.2, pp. 644-649

Klotz, S.; Chervin, J.-C.; Munsch, P. \& Le Marchand, G. (2009). Hydrostatic limits of 11 pressure transmitting media. Journal of Physics D: Applied Physics, Vol.42, pp. 075413-1-075413-7

Mei, Z.-G.; Shang, S.-L.; Wang, Y. \& Liu, Z.-K. (2009). Density-functional study of the thermodynamic properties and the pressure-temperature phase diagram of $\mathrm{Ti}$. Physical Review B, Vol.80, pp. 104116-1-104116-9

Ming, L.C.; Manghnani, M. \& Katahara, M. (1981). Phase-Transformations in the Ti-V System Under High-Pressure up to 25-GPa. Acta Metallurgica, Vol.29, No.3, pp. 479485

Pecker, S.; Eliezer, S.; Fisher, D.; Henis, Z. \& Zinamon, Z. (2005). A multiphase equation of state of three solid phases, liquid, and gas for titanium. Journal of Applied Physics, Vol.98, pp. 043516-1-043516-12 
Rosenberg, Z. \& Meybar, Y. (1981). Measurement of the Hugoniot curve of Ti-6Al-4V with commercial manganin gauges. Journal of Physics D: Applied Physics, Vol.14, pp. 261266

Tegner, B.E.; MacLeod, S.G.; Cynn, H.; Proctor, J.; Evans, W.J.; McMahon, M.I. \& Ackland, G.J. (2011). An Experimental and Theoretical Multi-Mbar Study of Ti-6Al-4V. Mater. Res. Soc. Symp. Proc., Vol.1369, Materials Research Society

Tegner, B.E.; MacLeod, S.G.; Cynn, H.; Proctor, J.; Evans, W.J. \& McMahon, M.I. (n.d.). Manuscript to be submitted.

Trinkle, D.R.; Hennig, R.G.; Srinivasan, S.G.; Hatch, D.M.; Jones, M.D.; Stokes, H.T.; Albers, R.C. \& Wilkins, J.W. (2003). New Mechanism for the a to $\omega$ Martensitic Transformation in Pure Titanium. Physical Review Letters, Vol.91, No.2, pp. 0257011-025701-4

Trinkle, D.R.; Hatch, D.M.; Stokes, H.T.; Hennig, R.G. \& Albers, R.C. (2005). Systematic pathway generation and sorting in martensitic transformations: Titanium $\alpha$ to $\omega$. Physical Review B, Vol.72, pp. 014105-1-014105-11

Trunin, R.F.; Simakov, G.V. \& Medvedev A.B. (1999). Compression of Titanium in Shock Waves. High Temperature, Vol.37, pp. 851-856

Velisavljevic, N. \& Chesnut, G.N. (2007). Direct hcp $\rightarrow$ bcc structural phase transition observed in titanium alloy at high pressure. Applied Physics Letters, Vol.91, pp. 101906-1-101906-3

Velisavljevic, N. (n.d.). Manuscript to be submitted.

Verma, A.K.; Modak, P.; Rao, R.S.; Godwal, B.K. \& Jeanloz, R. (2007). High-pressure phases of titanium: First-principles calculations. Physical Review B, Vol.75, pp. 014109-1014109-5

Vinet, P.; Ferrante, J.; Rose, J.H. \& Smith J.R. (1987). Compressibility of solids. Journal of Geophysical Research - Solid Earth and Planets, Vol.92, No.B9, pp. 9319-9325

Vohra, Y.K.; Sikka, S.K.; Vaidya, S.N. \& Chidambaram, R. (1977). Impurity Effects and Reaction-Kinetics of Pressure-Induced Alpha-Omega Transformation in Ti. Journal of Physics and Chemistry of Solids, Vol.38, No.11, pp. 1293-1296

Vohra, Y.K. (1979). Electronic basis for omega phase stability in group IV transition metals and alloys. Acta Metallurgica, Vol.27, No.10, pp. 1671-1674

Vohra, Y.K. \& Spencer, P.T. (2001). Novel $\gamma$-Phase of Titanium Metal at Megabar Pressures. Physical Review Letters, Vol.86, No.14, pp. 3068-3071

Xia, H.; Duclos, S.J.; Ruoff, A.L. \& Vohra, Y.K. (1990a). New High-Pressure Phase Transition in Zirconium Metal. Physical Review Letters, Vol.64, No.2, pp. 204-207

Xia, H.; Parthasarathy, H.L.; Vohra, Y.K. \& Ruoff, A.L. (1990b). Crystal structures of group IVa metals at ultrahigh pressures. Physical Review B, Vol.42, No.10, pp. 6736-6738

Zhang, J.; Zhao, Y.; Hixson, R.S.; Gray III, G.T.; Wang, L.; Utsumi, W.; Hiroyuki, S. \& Takanori, H. (2008). Experimental constraints on the phase diagram of titanium metal. Journal of Physics and Chemistry of Solids, Vol.69, pp. 25592563 
Zhou, T.; Aindow, M; Alpay, S.P.; Blackburn, M.J.; Wu, M.H. (2004). Pseudo-elastic deformation behavior in a Ti/Mo-based alloy. Scripta Materialia, Vol.50, pp. 343348 


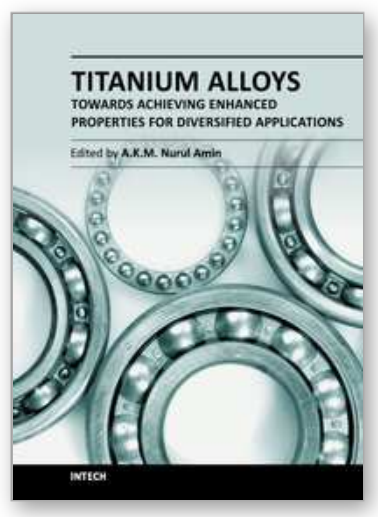

\author{
Titanium Alloys - Towards Achieving Enhanced Properties for \\ Diversified Applications
}

Edited by Dr. A.K.M. Nurul Amin

ISBN 978-953-51-0354-7

Hard cover, 228 pages

Publisher InTech

Published online 16, March, 2012

Published in print edition March, 2012

The first section of the book includes the following topics: fusion-based additive manufacturing (AM) processes of titanium alloys and their numerical modelling, mechanism of ?-case formation mechanism during investment casting of titanium, genesis of gas-containing defects in cast titanium products. Second section includes topics on behavior of the $(?+?)$ titanium alloys under extreme pressure and temperature conditions, hot and super plasticity of titanium (? + ?) alloys and some machinability aspects of titanium alloys in drilling. Finally, the third section includes topics on different surface treatment methods including nanotube-anodic layer formation on two phase titanium alloys in phosphoric acid for biomedical applications, chemico-thermal treatment of titanium alloys applying nitriding process for improving corrosion resistance of titanium alloys.

\title{
How to reference
}

In order to correctly reference this scholarly work, feel free to copy and paste the following:

Nenad Velisavljevic, Simon MacLeod and Hyunchae Cynn (2012). Titanium Alloys at Extreme Pressure Conditions, Titanium Alloys - Towards Achieving Enhanced Properties for Diversified Applications, Dr. A.K.M. Nurul Amin (Ed.), ISBN: 978-953-51-0354-7, InTech, Available from:

http://www.intechopen.com/books/titanium-alloys-towards-achieving-enhanced-properties-for-diversifiedapplications/titanium-alloys-at-extreme-pressure-conditions

\section{INTECH}

open science | open minds

\author{
InTech Europe \\ University Campus STeP Ri \\ Slavka Krautzeka 83/A \\ 51000 Rijeka, Croatia \\ Phone: +385 (51) 770447 \\ Fax: +385 (51) 686166 \\ www.intechopen.com
}

\author{
InTech China \\ Unit 405, Office Block, Hotel Equatorial Shanghai \\ No.65, Yan An Road (West), Shanghai, 200040, China \\ 中国上海市延安西路65号上海国际贵都大饭店办公楼405单元 \\ Phone: +86-21-62489820 \\ Fax: +86-21-62489821
}


(C) 2012 The Author(s). Licensee IntechOpen. This is an open access article distributed under the terms of the Creative Commons Attribution 3.0 License, which permits unrestricted use, distribution, and reproduction in any medium, provided the original work is properly cited. 